\title{
Registration of Preoperative CTA and Intraoperative Fluoroscopic Images for Assisting Aortic Stent Grafting
}

\author{
Hiroshi Imamura ${ }^{1}$, Noriaki Ida ${ }^{1}$, Naozo Sugimoto ${ }^{1}$, Shigeru Eiho ${ }^{1}$, \\ Shin-ichi Urayama ${ }^{2}$, Katsuya $\mathrm{Ueno}^{3}$, and Kanji Inoue ${ }^{3}$ \\ 1 Graduate School of Informatics, Kyoto University, \\ Uji-city, Kyoto, Japan 611-0011 \\ \{imamura,nrak, sugi, eiho\}@image.kuass.kyoto-u.ac.jp \\ 2 National Cardiovascular Center Research Institute, \\ Suita-city, Osaka, Japan 565-8565 \\ urayama@ri.ncvc.go.jp \\ 3 Takeda Hospital, Kyoto-city, Kyoto Japan 600-8558
}

\begin{abstract}
We investigated a registration method between preoperative 3D-CTA and intraoperative fluoroscopic images during intervention. Our final goal is assisting endovascular stent grafting for aortic aneurysm. In our method, DRR (Digitally Reconstructed Radiograph) are generated by voxel projection of 3D-CTA after extracting an aorta region. By increasing/decreasing CT value in the aorta region of CTA, DRR with/without contrast media injection are obtained. Subsequently we calculate matching measures between DRR and fluoroscopic images iteratively by changing imaging parameters. The most similar DRR to fluoroscopic image is selected. We investigated characteristics of several matching measures using simulated fluoroscopic images. From simulation results, we use M-estimator of residual in our method. From an application example to clinical data, registration was successfully applied by M-estimator of residual.
\end{abstract}

\section{Introduction}

Endovascular stent grafting is a minimal invasive treatment of aortic aneurysm [1. Currently 2D fluoroscopic image is used to visualize position of lesion or interventional device. Disadvantage in using fluoroscopic image is lack of information in 3D structure of the object. For discovering its information, registration of preoperative 3D CT angiogram (3D-CTA) and intraoperative 2D fluoroscopic image is useful. Therefore $3 \mathrm{D}-2 \mathrm{D}$ registration have been investigated by several groups[2] [3] [5].

As an application to intervention, Penney et al. developed new intensitybased similarity measure: pattern intensity and gradient difference [5. They reported both measures are robust to soft tissue deformation and presence of interventional device. In their method they use perspective model to project 3D CT image onto 2D fluoroscopic image. Therefore, this method has to search

T. Dohi and R. Kikinis (Eds.): MICCAI 2002, LNCS 2489, pp. 477-484 2002.

(C) Springer-Verlag Berlin Heidelberg 2002 
many imaging parameters (10 parameters) and it has problems of long calculation time and of narrow capture range. They reported that an estimation error in the direction perpendicular to the projection plane was significantly bigger than the other directions.

For intervention such as stent graft placement, CTA (with intra venous contrast injection) is usually taken as preoperative data and fluoroscopic image with/without contrast agent injection as intraoperative one. Penney et al. did not, however, investigate influence of contrast agent injection. In this paper we evaluate several intensity based measures including gradient difference. For reducing calculation time and having enough capture range, we use parallel projection model to project 3D CT image onto 2D fluoroscopic image and reduce the number of imaging parameters to 4 . By using parallel projection, it is impossible to estimate position in perpendicular direction to the projection plane. However, we think estimating rotation angles is much more important in aortic stent grafting than estimating position in the perpendicular direction. We also investigate influence of contrast agent both in preoperative and intraoperative images.

\section{Materials and Methods}

\subsection{Imaging Geometry and Image Specification}

Fig. 1 shows the coordinate system used in our method. We assume 4 imaging parameters, position $(x, z)$, angle (rotation, angulation). For a simulation study described in 2.3 and 3.1, CTA which covers wide area (from thorax and abdomen, matrix size : $512 \times 512 \times 313$ [pixel], voxel size : $0.664 \times 0.664 \times 1.250[\mathrm{~mm}])$ is used. Set of preoperative CTA (matrix size : $512 \times 512 \times 153$ [pixel], voxel size : $0.625 \times 0.625 \times 1.50[\mathrm{~mm}]$ ) and intraoperative fluoroscopic images (matrix size : $450 \times 450$ [pixel], pixel size : $0.390 \times 0.390[\mathrm{~mm}])$ of the same patient is also used in 2.4 and 3.2. These images were taken for placement of stent graft to abdominal aneurysm. Figures 2 and 3 show CTA images (axial, sagittal, and coronal slices), and Fig. 4 shows one of fluoroscopic images. Rectangle area on the fluoroscopic image is used for matching process described in the following subsection.

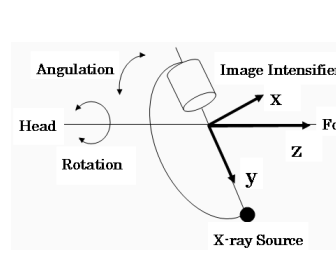

Fig. 1. Imaging ge- Fig ometry.
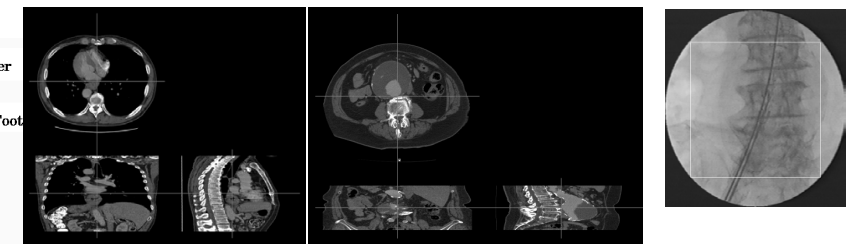

Fig. 4. Clinical ulation.
Fig. 3. CTA for clin- fluoroscopic imical application. age. 


\subsection{Method}

Generation of Digitally Reconstructed Radiograph. First, an aorta region is extracted from CTA using the extraction method which we previously developed [6]. Subsequently, by increasing/decreasing voxel value in aorta region, two kinds of $\mathrm{CT}$ data are produced. One is like a CT with intra aortic contrast injection, and the other is like a CT without contrast injection. Digitally reconstructed radiographs (DRR) of with/without contrast injection are, then, produced by parallel voxel projection of CT image with/without high contrasted aorta. By changing the imaging parameters (rotation and angulation in Fig. 1), a lot of DRRs are produced. The most similar DRR to a fluoroscopic image is searched among them.

Detecting Contrast Media Injection in Fluoroscopic Image. For detecting contrast media injection in fluoroscopic image, we accumulate pixel value inside ROI in fluoroscopic image (Fig. 4). If sum of pixel value is bigger/smaller than threshold, we estimate this fluoroscopic image is contrasted/non-contrasted and we use DRR with/without contrast media injection for registration.

\section{Matching Measures}

Residual. Residual between fluoroscopic image $\left(I_{\mathrm{ff}}\right)$ and DRR $\left(I_{\mathrm{DRR}}\right)$ are defined as follows. Residual can be calculated easily, but it depends on change of brightness and contrast of image. By using sequential similarity detection algorithm (SSDA) [7], calculation time to find a minimum value of this measure is able to be reduced significantly.

$$
R=\sum_{j=1}^{M} \sum_{i=1}^{N}\left|I_{\mathrm{fl}}(i, j)-I_{\mathrm{DRR}}(i, j)\right|
$$

M-estimator. Robust estimation is a statistic method which is robust to noise included only in one image. In interventional procedure, instruments such as stent or catheter are included only in fluoroscopic image, and there is a possibility that mismatch pair of image (contrasted/non-contrasted fluoroscopic image with non-contrasted/contrasted DRR) are used. M-estimator is one of the most popular criterions in robust estimation.

$$
M=\sum_{i, j} \frac{\left|I_{\mathrm{ff}}(i, j)-I_{\mathrm{DRR}}(i, j)\right|^{2}}{\sigma^{2}+\left|I_{\mathrm{f}}(i, j)-I_{\mathrm{DRR}}(i, j)\right|^{2}} \quad(\sigma: \text { constant })
$$

Gradient Difference. Penney et al. 5 presented a similarity measure for 3D$2 \mathrm{D}$ registration based on residual of gradient image: gradient difference. They show that it is robust to soft tissue deformation because low frequency components are already filtered out in gradient image. They show it is also robust to presence of linear high intensity region such as a stent or a catheter. 


$$
\begin{gathered}
G=\sum_{i, j} \frac{A_{v}}{A_{v}+\left\{I_{\mathrm{diff}}(i, j)\right\}^{2}}+\sum_{i, j} \frac{A_{h}}{A_{h}+\left\{I_{\mathrm{diffH}}(i, j)\right\}^{2}} \\
\left(A_{v}, A_{h}: \text { constants, } I_{\mathrm{diffV}}(i, j)=\frac{d I_{f l}}{d i}-s \frac{d I_{D R R}}{d i}\right. \\
\left.I_{\mathrm{diffH}}(i, j)=\frac{d I_{f l}}{d j}-s \frac{d I_{D R R}}{d j}\right)
\end{gathered}
$$

By the above formula, SSDA-like fast algorithm can not be utilized. However, this equation can be transformed to the following formula.

$$
\begin{aligned}
G & =\sum_{i, j}\left[1-\frac{\left\{I_{\mathrm{diffV}}(i, j)\right\}^{2}}{A_{v}+\left\{I_{\mathrm{diffV}}(i, j)\right\}^{2}}\right]+\sum_{i, j}\left[1-\frac{\left\{I_{\mathrm{diffH}}(i, j)\right\}^{2}}{A_{h}+\left\{I_{\mathrm{diffH}}(i, j)\right\}^{2}}\right] \\
& =\sum_{i, j}\{2\}-\left[\sum_{i, j} \frac{\left\{I_{\mathrm{diffV}}(i, j)\right\}^{2}}{A_{v}+\left\{I_{\mathrm{diff}}(i, j)\right\}^{2}}+\sum_{i, j} \frac{\left\{I_{\mathrm{diffH}}(i, j)\right\}^{2}}{A_{h}+\left\{I_{\mathrm{diffH}}(i, j)\right\}^{2}}\right]
\end{aligned}
$$

Maximizing $G$ gives the same result as minimizing the second term in the above formula. The second term is a distance measure, thus SSDA-like fast algorithm can be used for minimizing it. In this paper we minimize the second term which corresponds to an M-estimator of residual of gradient images.

We also investigated the following three measures, residual of gradient image, correlation coefficient, mutual information. We do not show result on these measures in this paper because result on residual of gradient image, cross correlation, and mutual information resembled gradient difference, residual, and M-estimator of residual respectively.

Optimization. We used multi-resolutional analysis for searching optimal imaging parameters. In this paper, we used triple resolutional data.

\subsection{Simulation Study}

For investigating a characteristics of matching measures, simulation study was performed. In this study, simulated fluoroscopic images were produced almost the same way for generating $\operatorname{DRR}(2.2 .1)$. However, here, perspective projection was used instead of parallel projection. S-shaped and pincushion distortion was also added on it (Fig. 5). Artificial line of high intensity is, then, added as simulated catheter. Figure 6 shows a finally obtained simulated fluoroscopic image. For investigating influence of rotation and angulation, we produced images from 3 kinds of imaging orientation (anterior, rotated, angulated images). In each case, both thoracic and abdominal images, and also with/without contrasted agent were generated. As a result, 12 fluoroscopic images are used in our study. By using these images and DRRs in Fig. 7, we investigated characteristics of matching measures described in 2.2. In this study we determined $A_{v}$ and $A_{h}$ for calculating gradient difference in the same way as described in Penney et.al[5]. 


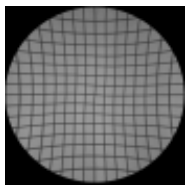

Fig. 5. Added geometric distortion to simulated fluoroscopy.

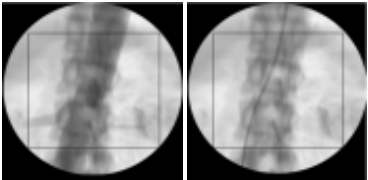

Fig. 6. Simulated fluoroscopic images (Left:contrast- Fig. 7. Generated DRR with ed, Right:non-constrasted). /without contrast agent injection.

Table 1. Average and standard deviation of estimation error.

(n)cFL :(Non-)Contrasted fluoroscopic image

(n)cDRR :(Non-)Contrasted DRR

\begin{tabular}{|c|c|c|c|c|c|}
\hline Measure & Pair of Image & Rotation [deg] & Angulation[deg] & $X[\mathrm{~mm}]$ & $Z[\mathrm{~mm}]$ \\
\hline \multirow{4}{*}{ residual } & cFL-cDRR & $-0.67 \pm 0.94$ & $0.00 \pm 1.16$ & $-0.22 \pm 0.50$ & $0.67 \pm 1.89$ \\
\hline & cFL-ncDRR & $0.67 \pm 8.72$ & $-6.33 \pm 7.90$ & $-13.79 \pm 23.01$ & $-1.33 \pm 12.70$ \\
\hline & ncFL-cDRR & $7.00 \pm 9.77$ & $3.67 \pm 6.70$ & $-13.12 \pm 21.86$ & $25.79 \pm 63.12$ \\
\hline & ncFL-ncDRR & $0.33 \pm 0.75$ & $0.67 \pm 2.49$ & $0.45 \pm 0.99$ & $4.00 \pm 3.75$ \\
\hline \multirow{4}{*}{$\begin{array}{c}\text { M-estimator } \\
\text { of residual }\end{array}$} & cFL-cDRR & $-0.33 \pm 0.82$ & $0.33 \pm 2.34$ & $0.45 \pm 1.38$ & $-0.45 \pm 2.85$ \\
\hline & cFL-ncDRR & $-1.00 \pm 5.33$ & $-1.67 \pm 9.59$ & $-5.34 \pm 6.64$ & $12.23 \pm 15.75$ \\
\hline & ncFL-cDRR & $-11.33 \pm 10.63$ & $-1.67 \pm 7.31$ & $-30.02 \pm 56.91$ & $53.36 \pm 34.58$ \\
\hline & ncFL-ncDRR & $-0.33 \pm 1.97$ & $1.00 \pm 2.45$ & $0.00 \pm 2.23$ & $-2.89 \pm 4.75$ \\
\hline \multirow{4}{*}{$\begin{array}{l}\text { gradient } \\
\text { difference }\end{array}$} & cFL-cDRR & $-2.00 \pm 0.00$ & $-1.00 \pm 1.67$ & $5.67 \pm 0.69$ & $0.00 \pm 3.13$ \\
\hline & cFL-ncDRR & $-8.00 \pm 8.67$ & $5.67 \pm 7.94$ & $-71.15 \pm 12.56$ & $40.24 \pm 51.99$ \\
\hline & ncFL-cDRR & $-3.00 \pm 3.52$ & $-4.33 \pm 7.74$ & $-27.57 \pm 39.87$ & $9.34 \pm 42.67$ \\
\hline & ncFL-ncDRR & $1.00 \pm 6.42$ & $3.67 \pm 8.34$ & $-10.89 \pm 21.48$ & $-4.89 \pm 5.30$ \\
\hline
\end{tabular}

\subsection{Application to Clinical Data}

We tested our algorithm to contrasted/non-contrasted clinical fluoroscopic image. First, we produced triple resolutional DRR. Subsequently we detected contrast media injection in fluoroscopic image and registered it with low resolutional DRR. For the lowest resolutional DRR and medium resolutional DRR, we used cross correlation as matching measure. For the highest resolutional data, we used M-estimator of residual as matching measure.

\section{Results}

\subsection{Simulation Study}

To examine accuracy of parameter estimation, we calculated average and standard deviation of estimation error for each parameter (Table 1).

The distributions of matching measures around peak point are calculated one dimensionally on a variable chosen. Residual, M-estimator of residual, and gradient difference are shown in Fig. 8 (a), (b), and (c) respectively. In these figures, one of imaging parameters is changed, other parameters are fixed with same value in the peak point. 

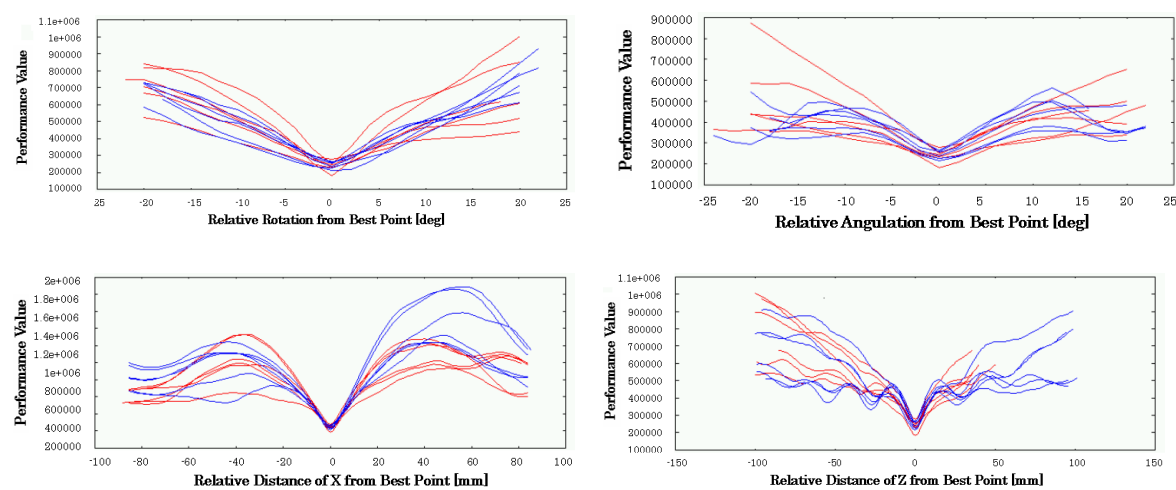

(a) Residual
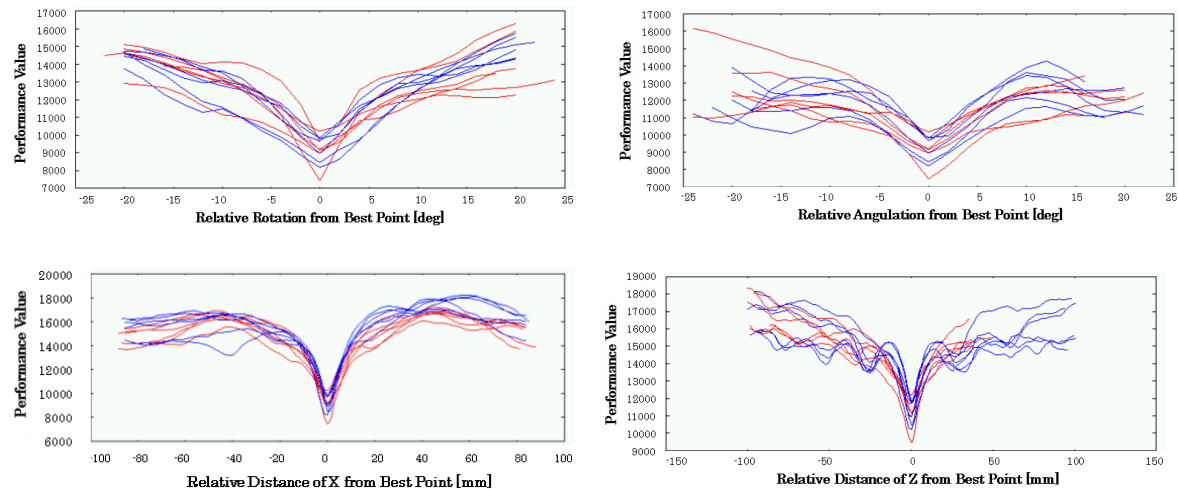

(b) M-estimator of residual
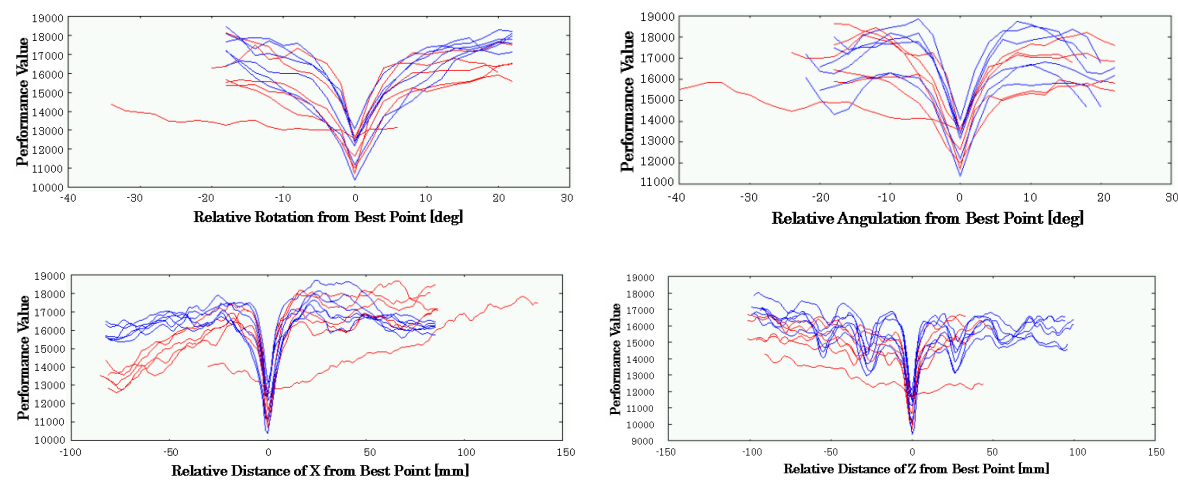

(c) Gradient difference (second term)

Fig. 8. One dimensional profile of matching measure distribution around peak point. 


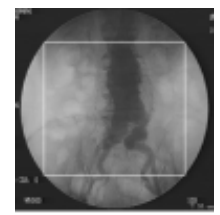

(a) contrasted
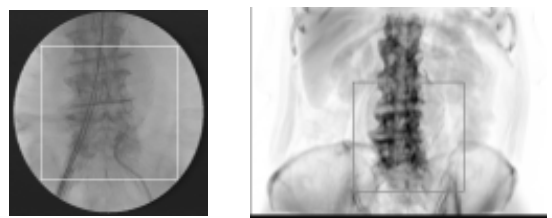

(b) non-contrasted

Fig. 9. Result of application to clinical data.

\subsection{Application to Clinical Data}

Experimental results of clinical fluoroscopic image with DRR are shown in Fig 9 (a) and (b). In these figures, left side image is original fluoroscopic image, and right side image is DRR with estimated imaging parameters.

\section{Discussion}

From Table 1, regarding influence of contrast injection, it was proved that a pair of contrasted fluoroscopic image with contrasted DRR is the best, non-contrasted fluoroscopic image with non-contrasted DRR is the second priority, and others are not good because average and standard deviation of estimation error are much bigger.

Standard deviation by gradient difference is much bigger than by other matching measures for non-contrasted fluoroscopic image with non-contrasted DRR case (ncFL-ncDRR). When edge of catheter in non-contrasted fluoroscopic image and edge of rib in non-contrasted DRR are matched, incorrect DRR is selected. Example of such a case is shown in Fig. 10. Edge of a rib and a catheter is indicated by inside of white ellipse region of left side and right side image.

Simulation study shows that residual of gradient, M-estimator of residual, gradient difference, mutual information have an enough sharp peak, but they have several local optimal points. On the other hand, residual and cross correlation have broad peak around ground truth. Therefore we use cross correlation to low resolutional data and M-estimator of residual to high resolutional data.

From clinical application, it seems that appropriate imaging parameters are estimated.

\section{Conclusion}

In this paper, we investigated a registration method between preoperative 3D CT angiography (3D-CTA) and intraoperative fluoroscopic images (with/without contrast injection) for assisting endovascular stent grafting. Especially we examined influence of contrast agent both in preoperative and intraoperative images. Simulation results and application to the clinical data show that M-estimator of residual is suitable as matching measure. 


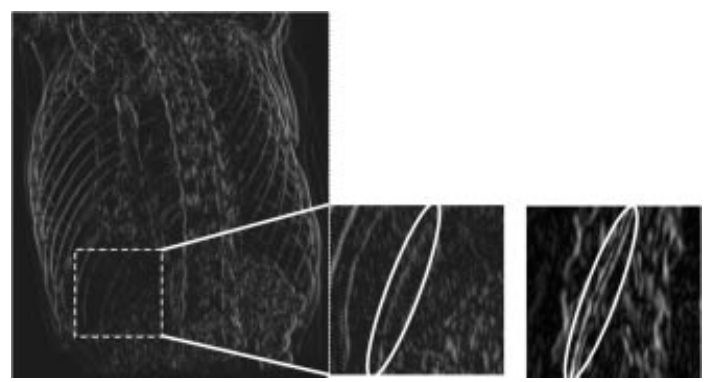

Fig. 10. Incorrectly selected differentiated DRR (Left, Middle) and differentiated simulated fluoroscopic image (Right).

\section{Acknowledgements}

This research is partially supported by Grant-in-Aid for Scientific Research (C)(2)(No.13680935) from Japan Society for the Promotion of Science(JSPS).

\section{References}

1. K. Inoue, H. Hosokawa, T. Iwase, M. Sato, Y. Yoshida, K. Ueno et al.: Aortic Arch Reconstruction by Transluminally Placed Endovascular Branched Stent Graft. Circulation 100 (1999) 316-321.

2. S. Lavallée and R. Szeliski: Recovering the Position and Orientation of Free-form Objects from Image Contours Using 3-D Distance Maps. IEEE Trans. PAMI 17 (1995) 378-390.

3. A. Guéziec, P. Kazanzides, B. Williamson, and R. H. Taylor: Anatomy Based Registration of CT-scan and X-ray Images for Guiding a Surgical Robot. IEEE Trans. Med. Imag. 17 (1998) 715-728.

4. L. Zöllei, E. Grimson, A. Norbash, W. Wells: 2D-3D Rigid Registration of X-Ray Fluoroscopy and CT Images Using Mutual Information and Sparsely Sampled Histogram Estimators. IEEE CVPR (2001).

5. G. P. Penney, J. Weese, J. A. Little, P. Desmedt, D. L. G. Hill, and D. J. Hawkes: A Comparison of Similarity Measures for Use in 2-D-3-D Medical Image Registration. IEEE Trans. Med.Imag. 17 (1998) 586-595.

6. H. Imamura, N. Sugimoto, S. Eiho, S. Urayama, K. Ueno, K. Inoue: Extraction and Quantitative Analyisis of Aneurysmal Aorta for Aiding Endovascular Stent Grafting. IEICE J84-D-II (2001) 2468-2476.

7. D. I. Barnea and H. F. Silverman: A class of algorithms for fast digital image registration. IEEE Trans. Comput. C-21 (1972) 179-186. 